\title{
Aortic penetration due to a fish bone: a case report
}

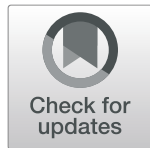

\author{
Daming Jiang ${ }^{1 *}$ (D, Yi Lu², Yigong Zhang ${ }^{1}$, Zhanglong Hu and Haifeng Cheng ${ }^{1}$
}

\begin{abstract}
Background: Aortoesophageal fistula (AEF) caused by an esophageal foreign body is a life-threatening crisis, with rapid progress and high mortality. The first case of AEF was reported in 1818, but the first successfully managed case was not until 1980. Although there have been some reports on this condition, in most cases, the aorta was invaded and corroded due to its adjacent relationship with the esophagus and subsequent mediastinitis. To date, few reports have described an aortic wall directly penetrated by a sharp foreign body, likely because this type of injury is extremely rare and most patients cannot receive timely treatment. Here, we present a rare case of a fish bone that directly pierced the aorta via the esophagus.
\end{abstract}

Case presentation: A 31-year-old female experienced poststernum swallowing pain after eating a meal of fish. Gastroscope showed a fishbone-like foreign body had penetrated the esophagus wall. Computed tomography revealed that the foreign body had directly pierced the aorta to form an AEF. Surgery was successfully performed to repair the aorta and esophagus. The postoperation and follow-up was uneventful.

Conclusions: For the treatment of foreign bodies in the esophagus, we should be alert of the possibility of AEFs. The effective management of AEFs requires early diagnosis and intervention, as well as long-term treatment and follow-up, which still has a long way to go.

Keywords: Aortoesophageal fistula, Esophageal foreign body, Esophageal perforation, Aortic penetration, Mediastinitis, Aortic infection, Thoracic endovascular aortic repair, Surgery, Case report

\section{Background}

Aortoesophageal fistula (AEF) caused by an esophageal foreign body is a life-threatening crisis, with rapid progress and high mortality [1]. Although there have been some reports on this condition, in most cases, the aorta was invaded and corroded due to its adjacent relationship with the esophagus and subsequent mediastinitis. Here, we present a rare case of a fish bone that directly pierced the aorta via the esophagus.

\footnotetext{
* Correspondence: to_jiangdm@zju.edu.cn

'Department of Cardiovascular Surgery, the Second Affiliated Hospital, Zhejiang University School of Medicine, 88 JieFang Road, Hangzhou, P.R. China 310009

Full list of author information is available at the end of the article
}

\section{Case presentation}

A 31-year-old female experienced poststernum swallowing pain for 2 days after eating a meal of fish. She denied any other symptoms such as fever or hematemesis. Gastroscope demonstrated a fishbone-like foreign body penetrating the esophagus wall $30 \mathrm{~cm}$ from the incisor (Fig. 1a). A further chest computed tomography (CT) scan revealed a sharp foreign body with a bone-like CT value just below the tracheal bifurcation, which had penetrated the thoracic aorta, resulting in mild pneumomediastinum and exudative changes around the esophagus (Fig. 1b). She was diagnosed with an AEF and referred for emergent open surgery.

The patient was placed in a right lateral position. A double-lumen tracheal tube was used to facilitate ventilation of the right lung during the operation. Extracorporeal circulation was established via femoral arterial and venous 


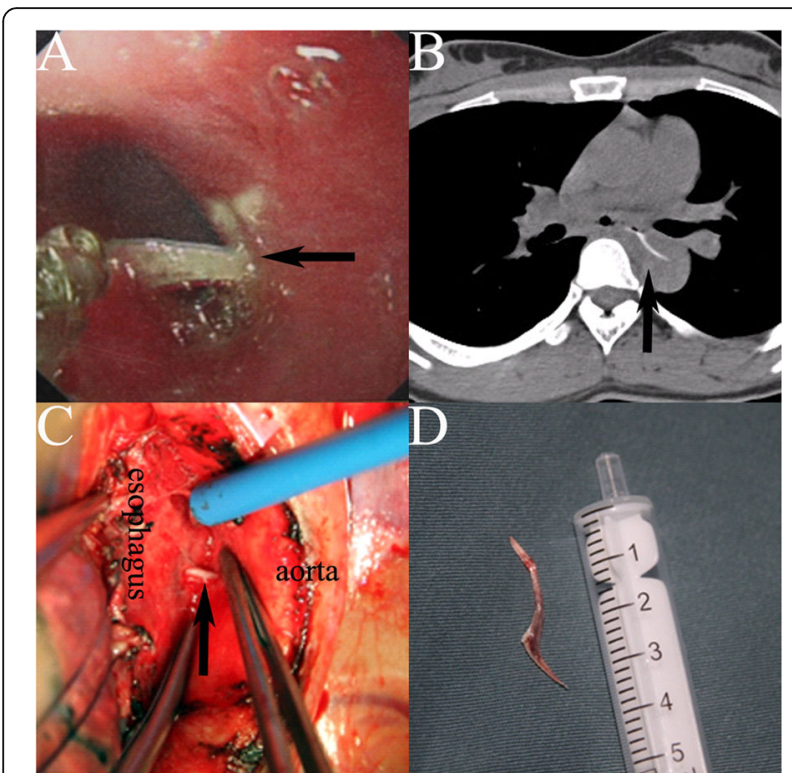

Fig. 1 a A typical picture of gastroscopy demonstrats the fish bone (arrow) piercing into the esophagus wall; b A CT shows a fish bone (arrow) in esophagus piercing directly into the thoracic aorta. c Fish bone (arrow) piercing from esophagus into the aorta (Intraoperative vision). d Fish bone after removal

cannulas. A left posterolateral thoracotomy through the fifth intercostal space was performed to gain access to the esophagus and descending aorta. The upper and lower normal aorta was isolated from the lesion area and blocking bands were set. Then, the mediastinal pleura was opened and the tissue adjacent to the lesion was separated. A long sharp fish bone emerged, which had penetrated the left esophageal wall approximately $2 \mathrm{~cm}$ below the tracheal carina level and pierced the descending aorta (Fig. 1c). The surrounding tissues had good vitality with mild edema. No obvious abscess was noted. After clamping the proximal and distal aorta, the contaminated tissue around the fish bone was removed and sent for culture (Fig. 2). After removal of the fish bone (Fig. 1d) and thorough debridement and irrigation with diluted polyvinylpyrrolidone-iodine and physiological saline solution, the injured aorta was repaired with 4-0 prolene suture, and the perforated esophagus was repaired with 3-0 absorbable coated Vicryl Plus antibacterial suture with the surrounding tissue flap. After repeated flushing, a drainage tube was placed in the mediastinum, and the chest was closed after the placement of another drainage tube in the chest cavity. Then, the patient was changed to the supine position and underwent laparoscopic jejunostomy. The patient recovered smoothly after the operation without complications and has lived freely for 5 years.

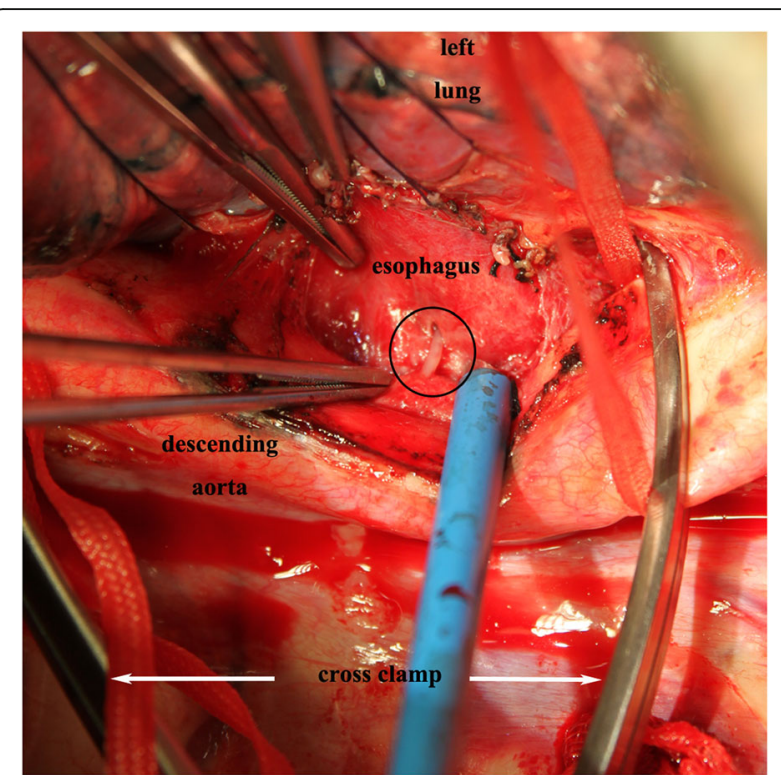

Fig. 2 Intraoperative picture shows the operative procedure (the circle presenting the fish bone)

\section{Discussion and conclusions}

$\mathrm{AEF}$ is a rare and dangerous condition, with a mortality rate between 40 and $60 \%[1,2]$. The first case of an AEF was reported in 1818, but the first successfully managed case was not until 1980 [3]. The common causes of AEFs include aortic aneurysms, esophageal foreign bodies and malignancy. To date, few reports have described an aortic wall directly penetrated by a sharp foreign body, likely because this type of injury is extremely rare and most patients cannot receive timely treatment.

AEF caused by an esophageal foreign body is often concealed. Patients may present with severe mediastinitis, eroding the adjacent aorta to form a pseudoaneurysm. Most patients have a clear history of an esophageal foreign body, accompanied by symptoms such as dysphagia, fever, and even Chiari triad (poststernum pain, sentinel hematemesis, and massive bleeding after an asymptomatic intermittent period). It is necessary to strive to transfer these patients to an experienced center within the golden therapeutic window (the period from sentinel hematemesis to massive bleeding, which may last several hours to months).

Once AEF is diagnosed, emergent surgery is needed $[1,4]$. There is currently no unified ideal treatment for AEF caused by an esophageal foreign body. The goals of surgery include controlling the bleeding, repairing the aorta, removing mediastinal infection, repairing the esophageal defect and reconstructing the digestive tract.

Whether thoracic endovascular aortic repair (TEVAR) can be used as a definitive treatment for AEFs is very controversial $[1,5]$. Many successful TEVAR cases have required additional thoracic surgeries, such as thoracoscopic 
or open surgery for mediastinal debridement and drainage, and esophageal repair $[4,6]$. Most of the available evidence has shown that TEVAR is the first choice for rescuing patients with unstable hemodynamic parameters $[1,5]$. Subsequent open surgery is recommended within 1 week after TEVAR to avoid infection of the implanted stent.

Compared with TEVAR, aortic repair with open surgery is more effective [1]. According to the size of the aortic defect, patch repair or artificial graft replacement is selected. Direct suture is suitable for small aortic perforations with no obvious signs of infection. It is recommended that a left thoracotomy be performed using extracorporeal circulation, such as femoral arterial and venous bypass, left heart bypass, etc. If the aortic involvement is wide and the arch branches are severely affected, deep hypothermia arrest may be needed [7]. After exposure of the aorta, the range and extent of the involving aorta should be carefully evaluated. The infected aorta around the fistula should be debrided and removed to eliminate potential reinfection. Compared with artificial graft, homograft has certain advantages in infectious aortic replacement [5, 7].

Esophageal perforation caused by a foreign body generally requires surgical repair [8]. There is currently no consensus on direct esophageal repair or partial resection. The surgical plan depends on the location of the perforation, the characteristics of the foreign body, and the severity of the mediastinitis [8]. Direct repair is suitable for patients with early detection of esophageal perforation, esophageal infection with few necrotic tissues, and limited mediastinal inflammation [8]. Postoperative swallowing function is better with direct repair than with resection. However, most esophageal perforations are not detected until the mediastinitis is obvious. As a result, more surgeons choose to perform esophagectomy to reduce the risk of reinfection; they also tend to reconstruct the digestive tract in stages and make the anastomosis on the neck.

The pedicled omentum can be used to wrap the repaired aorta or esophagus and fill the cavity after debridement. It has a great advantage in the treatment of infectious diseases such as AEFs, because it can isolate the infection and significantly reduce reinfection and mortality $[5,9,10]$. The pedicled intercostal muscle flap [11] and sternocleidomastoid muscle flap [12] have similar effects.

Although the clinical management of AEFs has made great progress in the past few decades, the postoperative mortality is still very high. We consider that the AEFs caused by an esophageal foreign body rarely directly penetrate the aorta and often has mild mediastinitis; thus, we directly repaired the aorta and esophagus without pedicled omentum flap wrapping. In addition, patients with AEFs generally need to fast for a long period of time, and jejunostomy has obvious advantages, with a lower incidence of long-term complications; this procedure is now used routinely at our center. Our therapeutic strategy resulted in satisfactory short- and long-term outcomes.

In conclusion, for the treatment of foreign bodies in the esophagus, we should be alerted to the possibility of AEFs. The effective management of AEFs requires early diagnosis and early intervention, as well as long-term treatment and follow-up, which still has a long way to go.

\section{Abbreviations}

AEF: Aortoesophageal fistula; CT: Computed tomography; TEVAR: Thoracic endovascular aortic repair

\section{Acknowledgements}

Not applicable.

\section{Authors' contributions}

DMJ and YL wrote the manuscript. YGZ and ZLH obtained the image data. All authors were involved in the treatment of the patient. All authors read and approved the final written manuscript.

\section{Funding}

Not applicable.

Availability of data and materials

The datasets of the current study are available from the corresponding author upon reasonable request.

Ethics approval and consent to participate

The ethics committee of the Second Affiliated Hospital of Zhejiang University approved the study.

\section{Consent for publication}

Consent for publication is obtained from the patient.

\section{Competing interests}

The authors declare that they have no competing interests.

\section{Author details}

${ }^{1}$ Department of Cardiovascular Surgery, the Second Affiliated Hospital, Zhejiang University School of Medicine, 88 JieFang Road, Hangzhou, P.R. China 310009. ${ }^{2}$ Department of Cardiology, the Second Affiliated Hospital, Zhejiang University School of Medicine, Hangzhou, China.

Received: 1 June 2020 Accepted: 21 September 2020

Published online: 02 October 2020

\section{References}

1. Takeno S, Ishii H, Nanashima A, Nakamura K. Aortoesophageal fistula: review of trends in the last decade. Surg Today. 2019. https://doi.org/10.1007/ s00595-019-01937-z.

2. Göbölös L, Miskolczi S, Pousios D, et al. Management options for aortooesophageal fistula: case histories and review of the literature. Perfusion. 2013 Jul;28(4):286-90.

3. Ctercteko G, Mok CK. Aorta-esophageal fistula induced by a foreign body: the first recorded survival. J Thorac Cardiovasc Surg. 1980 Aug;80(2):233-5.

4. Chen X, Li J, Chen J, et al. A combined minimally invasive approach for the treatment of aortoesophageal fistula caused by the ingestion of a chicken bone: case report and literature review. Clinics (Sao Paulo). 2012;67(2):195-7.

5. Kelly SL, Peters P, Ogg MJ, Li A, Smithers BM. Successful management of an aortoesophageal fistula caused by a fish bone--case report and review of literature. J Cardiothorac Surg. 2009 May 8;4:21.

6. Wei $Y$, Chen L, Wang Y, Yu D, Peng J, Xu J. Proposed management protocol for ingested esophageal foreign body and aortoesophageal fistula: a singlecenter experience. Int J Clin Exp Med. 2015;8(1):607-15. 
7. Sica GS, Djapardy V, Westaby S, Maynard ND. Diagnosis and management of aortoesophageal fistula caused by a foreign body. Ann Thorac Surg. 2004 Jun; $77(6): 2217-8$

8. Triadafilopoulos G, Roorda A, Akiyama J. Update on foreign bodies in the esophagus: diagnosis and management. Curr Gastroenterol Rep. 2013 Apr; 15(4):317.

9. Zhu Y, MacArthur JW, Lui NS, Lee AM. Surgical Management for Aortoesophageal Fistula After Endovascular Aortic Repair. Ann Thorac Surg. 2020;109(5):1611-13

10. LeMaire SA, Coselli JS. Options for managing infected ascending aortic grafts. J Thorac Cardiovasc Surg. 2007 Oct;134(4):839-43.

11. Assink J, Vierhout BP, Snellen JP, et al. Emergency endovascular repair of an aortoesophageal fistula caused by a foreign body. J Endovasc Ther. 2005 Feb;12(1):129-33.

12. Venara A, Hamdi S, Desolneux G, et al. Long-term successful management of an aortoesophageal fistula secondary to the ingestion of a bone. Presse Med. 2012 May;41(5):543-6.

\section{Publisher's Note}

Springer Nature remains neutral with regard to jurisdictional claims in published maps and institutional affiliations.

Ready to submit your research? Choose BMC and benefit from:

- fast, convenient online submission

- thorough peer review by experienced researchers in your field

- rapid publication on acceptance

- support for research data, including large and complex data types

- gold Open Access which fosters wider collaboration and increased citations

- maximum visibility for your research: over $100 \mathrm{M}$ website views per year

At BMC, research is always in progress.

Learn more biomedcentral.com/submissions 